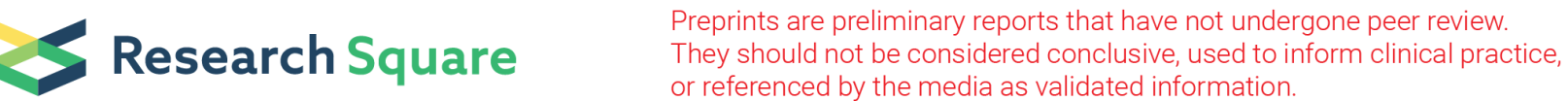

\section{Variation in volumes and characteristics of ENT emergency visits during COVID-19 pandemic. Where are the patients?}

\section{Marilia Sapoutzi ( $\square$ marilia.sapountzi@gmail.com )}

National \& Kapodistrian University of Athens, School of Medicine, 2nd Otolaryngology Department, "Attikon" University Hospital, Athens, Greece.

\section{Giorgos Sideris}

National \& Kapodistrian University of Athens, School of Medicine, 2nd Otolaryngology Department, "Attikon" University Hospital, Athens, Greece

\section{Eleni Boumpa}

National \& Kapodistrian University of Athens, School of Medicine, 2nd Otolaryngology Department, "Attikon" University Hospital, Athens, Greece

\section{Nikolaos Papadimitriou}

National \& Kapodistrian University of Athens, School of Medicine, 2nd Otolaryngology Department, "Attikon" University Hospital, Athens, Greece

\section{Thomas Nikolopoulos}

National \& Kapodistrian University of Athens, School of Medicine, 2nd Otolaryngology Department, "Attikon" University Hospital, Athens, Greece

\section{Alexander Delides}

National \& Kapodistrian University of Athens, School of Medicine, 2nd Otolaryngology Department, "Attikon" University Hospital, Athens, Greece

\section{Short Report}

Keywords: COVID-19, SARS-CoV-2, Otolaryngology, Emergency medicine, ENT

Posted Date: June 19th, 2020

DOI: https://doi.org/10.21203/rs.3.rs-35778/v1

License: (c) (i) This work is licensed under a Creative Commons Attribution 4.0 International License. Read Full License 


\section{Abstract}

Purpose: The aim of this study is to analyze patterns and volumes of patients visiting ENT emergency departments during periods of community lockdowns given the newness of this situation and to assess the variation in numbers of visits and examine whether their reduction equally affects all categories of visit causation or whether it is limited to diseases not considered as emergencies.

Methods: A retrospective descriptive study was conducted on prospectively collected registry data on patients of all age groups visited the emergency ENT department during the period of nationwide lockdown. Patients were grouped in 9 categories, according to the cause of visit the ENT emergency department.

Results: A total of 201 patients visited the ENT emergency department. The retrospective analysis of the data during the same period in the years 2013 to 2019 showed a reduction of $73 \%$ in total visits (mean total visits number between 2013 to 2019 was $745.29 \pm 20.0143$ ). A statistically significant reduction in visitation was found in all categories studied with the exception of foreign body ingestion - aspiration.

Conclusion: Patients with General ENT symptoms, mild epistaxis cases, otology cases, vertigo cases, uncomplicated infectious cases showed a statistically significant reduction in numbers and were treated empirically. Swallowing foreign body cases did not show statistically significant reduction. Limitation of movement and the lockdown itself, led to reduction of trauma cases. Surprisingly, there was also a reduction in oncology cases and an increase in numbers of these cases can be expected in the near future.

\section{Introduction}

In early 2020, the World Health Organization declared the outbreak of the disease caused by a new variant of Coronavirus SARS-Cov2, now called COVID-19 as a global pandemic. In Greece, Governmentled lockdown and mandatory quarantine were implemented on March 10,2020.

In Athens metropolitan area all hospitals including tertiary university centers are open for all emergency cases on 4-day intervals. Visits include referrals by primary health care physicians, emergency medical services as well as self-referral patients. A socialized medical system covers patient's hospital expenses for all emergencies. During the COVID-19 outbreak thirteen hospitals have been designated as reference hospitals to deal with COVID-19 cases [1]. "Attikon" University Hospital, the largest University hospital in the Attika area, was declared a COVID-19 referral center but emergency services were not suspended. Regarding Otolaryngology services, elective outpatient clinics as well as elective surgeries were suspended, as were all sub-specialty clinics. All emergency ENT services remained open to the public.

During quarantine, all private medical office services including private hospitals in the Athens area were limited to emergency-only cases and telephone-advice was given by primary care doctors. All civilians 
needing any emergency care were free to access all emergency hospitals, and an automated text messaging service was used as a travel permit in the city.

It is known that many patients may be avoiding hospitals to minimize risk of SARS-CoV-2 infection [2]. Although, little is known about the patterns and volumes of patients visiting ENT emergency departments during periods of community lockdowns given the newness of this situation. The aim of this study is to assess the variation in numbers of visits and examine whether their reduction equally affects all categories of visit causation or whether it is limited to diseases not considered as emergencies.

\section{Methods}

A retrospective descriptive study was conducted on prospectively collected registry data on patients of all age groups visited the emergency ENT department between March 10 and April 30 from the year 2013 onwards. This is the period of nationwide lockdown and limitation of movement in Greece, during the COVID-19 pandemic. Patients were grouped in 9 categories, according to the cause of visit: general ENT, foreign body ingestion-aspiration, trauma, vertigo, epistaxis, otology, emergency head and neck cancer (including hemorrhage and airway compromise), deep neck infections including supraglottitis and sialadenitis as well as insecure airway cases.

Statistical analysis was performed using the IBM SPSS Statistics 25.0 software (SPSS Inc., Chicago, IL, USA) and Microsoft Office Excel 2019 (Microsoft Corporation, Redmond, WA). One-sample t-test was used for comparison of means between groups with current visits. $P$ values $<.05$ were considered statistically significant, whereas the test of heterogeneity used a level of $0.10 \mathrm{~s}$

\section{Results}

A total of 201 patients visited the ENT emergency department during the period between March 10 and April 30, 2020 when the national lockdown was in effect. Grouping these patients in categories according to the cause of visit resulted in: 45 patients with general ENT symptoms, 20 with epistaxis, 45 otology , 12 deep neck infections, 26 with vertigo, 10 head and neck cancer cases, 5 with insecure airway, 13 with foreign body and 25 trauma cases. The retrospective analysis of the data during the same period in the years 2013 to 2019 showed a reduction of $73 \%$ in total visits (mean total visits number between 2013 to 2019 was $745.29 \pm 20.0143$ ). A statistically significant reduction in visitation was found in all categories studied with the exception of foreign body ingestion - aspiration (Table 1). Most noticeable reductions were evident in the General ENT diagnosis (84\% reduction, $\mathrm{p}<.00001)$ and the lowest reduction occurred in the Foreign Body diagnosis (46.4\% reduction, $\mathrm{p}=0.058376$ ).

\section{Discussion}

This study has revealed significant reductions in the overall number of all visits in an ENT emergency department during the lockdown period. Available unpublished data of the Athens area show a reduction 
in ENT visits to other health care facilities as well, including public and private hospitals.

The total reduction in the number of ENT emergency visits by $73 \%$ during the COVID-19 pandemic have many explainations. The fact that not only true emergencies visit ENT emergency departments due to the length of waitlists to access outpatient care has been also reported by other authors with similar health care systems, such as Italy [3].

In our study the greatest reductions were seen in general ENT cases and infections that do not need hospitalization. This suggests that most of the general ENT cases that present to the ENT emergency department of a tertiary hospital due to the referral pattern in this country could/should be treated by a primary or secondary health care facility. Furthermore, an overall reduction of other upper respiratory infections should me attributed to the lockdown including school closure.

Telephone advice was used during the lockdown period by most primary care physicians as well as private office-based Otolaryngologists. Since a reduction of deep neck infections was also noted, this could imply that early advice and treatment strategies used by the community health care professionals including local pharmacists was in fact effective. It is established that self-medication by patients and antimicrobial consumption are high in Greece. Moreover, there is also a dispensing of antibiotics by pharmacies without prescription [4].

The second greater reduction was seen in otology cases, suggesting its cause to be similar to general ENT cases.

Patients with vertigo presumably remained at home waiting for their symptoms to recess.

In regard to trauma cases, it is safe to presume that the lockdown itself has led to a reduction of these cases. Reports reveal that there is a significant decrease in visits to emergency trauma departments [5]. Other authors have reported the lower volumes of trauma and injury cases caused by fight, vehicle, ladder accidents or incidents at work, school and sport [6].

We can assume that mild epistaxis incidents could be and were managed by the patients themselves. It is known that while the great majority of epistaxis are limited in severity and duration, only about $6 \%$ will seek medical attention. Interventions for nosebleeds range from self-treatment and home remedies to more intensive procedural interventions in medical offices, emergency departments, hospitals, and operating rooms. Epistaxis has been estimated to account for $0.5 \%$ of all emergency department visits and up to one-third of all otolaryngology-related emergency department encounters. Inpatient hospitalization for aggressive treatment of severe nosebleeds has been reported in $0.2 \%$ of patients with nosebleeds [7].

On the other hand, the least reduction occurred in foreign body cases; this does not come as a surprise since most foreign body ingestion incidents happen in the home and cannot be self-treated. 
Surprisingly, there was also a reduction in oncology cases, including insecure airway and an increase in numbers of these cases or cases of inoperable or advanced stage cancer, may be expected in the near future. Oncologic and insecure airway cases, although found to have a statistically significant reduction in volume of visits, are traditionally considered real emergency cases and this reduction was not expected. However, a significant increase in the visitation numbers of these cases is expected in the period following the lift of the national lockdown, due to the large numbers of undiagnosed/untreated oncologic cases. It is mentioned that even oncology cases, although prioritized, are sometimes impacted to limit necessity of ICU or tracheostomy [8,9]. Patients had to balance the risk of diagnosis/treatment delay versus harm from COVID-19 disease. This resulted in a change of the patient's perception as to what is considered an emergency and should seek treatment in an emergency department of a tertiary hospital. We should keep in mind, though, that many of the cases that did not present to the emergency department during the COVID-19 pandemic could very well present at a later stage with increased severity.

The impact of SARS-CoV-2 pandemic on otolaryngology diagnostic and surgical activities is still unknown, as well as the effects on future general health care strategy and hospital organization. The current situation has caused a drastic reduction of the routine activity of otolaryngology units, with a dramatic impact on the service offered to patients [10]. The reduction in admissions observed should raise serious concerns about the well-being and health outcomes of patients who are not receiving inpatient care for conditions that require emergency treatment [11].

One of the greatest enemies is the consequences of hospitalizations' reduction for conditions requiring timely treatment. One of the greatest friends is to find the way to earn the patients back.

\section{Conclusion}

Novel Coronavirus Pandemic and the national lockdown along with movement limitation that occurred, caused a reduction in general numbers of visits to the ENT emergency department of our Hospital compared with the same period during the previous years. Patients with General ENT symptoms, mild epistaxis cases, otology cases, vertigo cases, uncomplicated infectious cases showed a statistically significant reduction in numbers and were treated empirically. Swallowing foreign body cases did not show statistically significant reduction. Limitation of movement and the lockdown itself, led to reduction of trauma cases. Surprisingly, there was also a reduction in oncology cases and an increase in numbers of these cases can be expected in the near future. This paper indicates the necessity of further studying the effects of the lockdown and the need to stay vigilant after the lockdown period.

\section{Declarations}

\section{Compliance with ethical standards}

Conflict of interest: The authors declare that they have no confict of interest. 
Ethical approval: This article does not contain any studies with human participants or animals performed by any of the authors.

Informed consent: Not applicable.

\section{Funding: None}

The authors declare that they have no known competing financial interests, no confict of interest or personal relationships that could have appeared to influence the work reported in this paper. This is a retrospective chart review study that was approved by the Institutional Review Board and ethics committee of the Attikon University Hospital - number of approval IRB 337/19-6-20. Ethics committee approval (IRB 337/19-6-20) includes the patient's informed consent wherever applicable according to hospital's guidelines.

\section{References}

[1]Giannopoulou I, Tsobanoglou GO (2020) COVID-19 pandemic: Challenges and opportunities for the Greek Health Care System. Ir J Psychol Med 14:1-9. https://doi.org/10.1017/ipm.2020.35

[2] Rosenbaum L (2020) The untold toll-the pandemic's effects on patients without Covid-19. N Engl J Med. https://doi.org/10.1056/NEJMms2009984

[3] Gelardi M, lannuzzi L, Trecca EMC et al (2020) COVID-19: What Happened to All of the Otolaryngology Emergencies? Eur Arch Otorhinolaryngol. https://doi.org/10.1007/s00405-020-06046-z.

[4] Karakonstantis S, Kalemaki D (2019) Antimicrobial overuse and misuse in the community in Greece and link to antimicrobial resistance using methicillin-resistant S. aureus as an example. J Infect Public Health 12(4):460-464. https://doi.org/10.1016/j.jiph.2019.03.017.

[5] Thornton J (2020) Covid-19: A\&E visits in England fall by 25\% in week after lockdown. BMJ. https://doi.org/10.1136/bmj.m1401

[6] Christey G, Amey J, Campbell A, Smith A (2020) Variation in volumes and characteristics of trauma patients admitted to a level one trauma centre during national level 4 lockdown for COVID-19 in New Zealand. N Z Med J 133(1513):81-88.

[7] Tunkel et al (2020) Clinical Practice Guideline: Nosebleed (Epistaxis) Executive Summary. Otolaryngol Head Neck Surg 162(1):8-25. https://doi.org/10.1177/019459981988995

[8] Simon F (2020) Ethical questions related to Covid-19 and ENT practice. Eur Ann Otorhinolaryngol Head Neck Dis 137(3):155-156. https://doi.org/10.1016/j.anorl.2020.04.009.

[9] Soldatova L, Rassekh CH, Baloch ZW et al (2020) Salivary Gland Disease in the Era of COVID-19 Pandemic. Head Neck 42(6):1339-1343. https://doi.org/10.1002/hed.26210. 
[10] Ralli M, Greco A, De Vincentiis M (2020) The Effects of the COVID-19/SARS-CoV-2 Pandemic Outbreak on Otolaryngology Activity in Italy. Ear Nose Throat $\mathrm{J}$.

https://doi.org/10.1177/0145561320923893.

[11] Baum A, Schwartz MD (2020) Admissions to Veterans Affairs Hospitals for Emergency Conditions During the COVID-19 Pandemic. JAMA. https://doi.org/10.1001/jama.2020.9972

\section{Tables}

Table 1: Statistical analysis and comparison of admission volumes depending of the cause of admission for same time periods in the years 2013 to 2019

\begin{tabular}{|r|c|c|c|c|c|}
\hline & 2020 & \multicolumn{3}{|c|}{$2013-2019$} & P value \\
\hline & $\mathrm{n}$ & Mean & Min-Max 95\%CI of Mean & \\
\hline Total & 201 & 745.3 & 726.8 & 763.8 & $<0.0001$ \\
\hline General ENT & 45 & 285.0 & 272.5 & 297.5 & $<0.0001$ \\
\hline Epistaxis & 20 & 58.6 & 44.11 & 73.04 & $<0.0001$ \\
\hline Otology & 45 & 194.4 & 180.8 & 208 & $<0.0001$ \\
\hline Deep infections & 12 & 28.0 & 22.03 & 33.97 & $<0.0001$ \\
\hline Vertigo & 26 & 76.9 & 66.8 & 86.91 & $<0.0001$ \\
\hline Oncology & 10 & 20.6 & 18.38 & 22.76 & $<0.0001$ \\
\hline Airway & 5 & 14.7 & 12.89 & 16.54 & 0.058 \\
\hline Foreign body & 13 & 20.4 & 12.64 & 28.22 & $<0.0001$ \\
\hline & & & & & \\
\hline
\end{tabular}

